\title{
Cell-Free Systems: A Proving Ground for Rational Biodesign
}

\author{
Nadanai Laohakunakorn * \\ School of Biological Sciences, Institute of Quantitative Biology, Biochemistry, and Biotechnology, University of Edinburgh, \\ Edinburgh, United Kingdom
}

OPEN ACCESS

Edited by:

Thomas Edward Gorochowski, University of Bristol, United Kingdom

Reviewed by:

Manish Kushwaha, INRA UMR1319 Microbiologie de l'Alimentation au Service de la Santé,

France

Borkowski Olivier, Institut Pasteur, France

*Correspondence:

Nadanai Laohakunakorn nadanai.laohakunakorn@ed.ac.uk

Specialty section: This article was submitted to

Synthetic Biology,

a section of the journal Frontiers in Bioengineering and Biotechnology

Received: 30 April 2020 Accepted: 22 June 2020 Published: 24 July 2020

Citation:

Laohakunakorn N (2020) Cell-Free Systems: A Proving Ground for Rational Biodesign

Front. Bioeng. Biotechnol. 8:788. doi: 10.3389/fbioe.2020.00788
Cell-free gene expression systems present an alternative approach to synthetic biology, where biological gene expression is harnessed inside non-living, in vitro biochemical reactions. Taking advantage of a plethora of recent experimental innovations, they easily overcome certain challenges for computer-aided biological design. For instance, their open nature renders all their components directly accessible, greatly facilitating model construction and validation. At the same time, these systems present their own unique difficulties, such as limited reaction lifetimes and lack of homeostasis. In this Perspective, I propose that cell-free systems are an ideal proving ground to test rational biodesign strategies, as demonstrated by a small but growing number of examples of model-guided, forward engineered cell-free biosystems. It is likely that advances gained from this approach will contribute to our efforts to more reliably and systematically engineer both cell-free as well as living cellular systems for useful applications.

Keywords: cell-free synthetic biology, cell-free protein synthesis, in vitro transcription translation, model-guided design, rational design

\section{INTRODUCTION}

A basic aim of synthetic biology is to design and construct biological systems which perform a given function. An extension of this, inspired by common engineering practice, is to additionally demand that the systems perform robustly, predictably, and with quantitative precision. Some practitioners of synthetic biology explicitly adopt the conventional engineering approach of rational design, where a system is constructed predictively (Endy, 2005; Heinemann and Panke, 2006). In contrast to non-biological engineering, synthetic biological systems are also open to the possibility of evolutionary design (Arnold, 1998), where function is obtained through directed evolutionary screens. It is still an open question as to whether or not a purely rational engineering approach can ultimately be successfully applied to engineer complex biomolecular systems (Davies, 2019).

A fully rational approach adopts all conventional engineering principles, such as standardization and quantitative characterization of parts, mathematical models to describe their behavior, and abstraction which allows hierarchical assembly of parts into modules, subsystems, and systems (Endy, 2005; Arkin, 2008; Canton et al., 2008). For any system of non-trivial complexity, this approach relies on computational methods to enable predictive design (MacDonald et al., 2011).

To a large extent, strict adherence to this approach has not yet been widely successful in synthetic biology, with a few notable exceptions (e.g., Nielsen et al., 2016). Typically, biodesign involves multiple iterations through a so-called "design-build-test-learn" (DBTL) cycle. While eventually a functioning system is produced, the path to get there is not directly through predictive design, but rather informed trial-and-error. The current necessity of DBTL cycles is due to partly to the fact that the complexity associated with biomolecular systems eludes simplifying black-box approximations common in other physical scenarios. Additionally, interrogating biosystems with controlled inputs 
and perturbations is difficult, and system parameters can be context-dependent and varying. However, with the emergence of high-throughput automation and biofoundries (Hillson et al., 2019), the promise is that DBTL efforts will ultimately enable fully predictive, rational biodesign.

Cell-free systems (Garenne and Noireaux, 2019) can contribute in several ways to improve the design process of synthetic biological systems, which span scales from the molecular (genetic regulatory elements, proteins, enzymes), to the systemic (gene regulatory and metabolic networks), and all the way to the extracellular levels (synthetic cells, communication, self-assembly). First, they can accelerate DBTL cycles through rapid prototyping (Chappell et al., 2013; Niederholtmeyer et al., 2015; Takahashi et al., 2015). Second, they can be used efficiently for in vitro directed evolution (ContrerasLlano and Tan, 2018). In this Perspective I would like to focus on a third contribution, and suggest that they offer an ideal proving ground to test the approach of rational computer-aided biodesign as applied to biomolecular systems (Figure 1). In particular, they present features which overcome some of the difficulties associated with engineering living cells, and so can be used to more easily develop and calibrate mechanistic models, as well as generate sufficient data for machine learning approaches.

To understand their strengths and weaknesses in the context of synthetic biology, it is first important to consider the differences between cell-free and living cellular systems.

\section{BIOPHYSICAL DIFFERENCES BETWEEN CELL-FREE AND CELLULAR SYSTEMS}

Today, cell-free technology generally refers to cell-free protein synthesis (CFPS), which rests on the foundational processes of in vitro transcription and translation (Silverman et al., 2019a; Laohakunakorn et al., 2020). Strictly speaking, CFPS belongs to the much broader field of in vitro reconstitution, which consists of recapitulating biological processes outside of the living cell. This involves combining relevant enzymes (either purified or extracted in crude cellular lysate) with a reaction mixture containing substrates, cofactors, and specific ionic and $\mathrm{pH}$ conditions. Constructing such a reaction isolates specific biological processes, and has historically served as a key approach to elucidate molecular biological mechanisms, including deciphering the genetic code itself (Nirenberg and Matthaei, 1961; Zubay, 1973). While this article will focus predominantly on bacterial cell-free systems due to their current widespread use, cell-free systems have also been successfully prepared from a number of prokaryotic and eukaryotic organisms (Perez et al., 2016).

In addition to developing fundamental understanding, this approach also enables technological applications: examples of these are the use of CFPS to carry out in vitro biomanufacturing, where the production of exogenous protein is advantageously decoupled from cellular growth (Karim and Jewett, 2018; Gregorio et al., 2019); and biosensing, where robust, lyophilized cell-free gene circuits can be activated and used to detect environmental contaminants and pathogens directly in the field (Pardee et al., 2014).

One predominant viewpoint of cell-free systems is that they are cellular mimics. The crude cellular lysate is a representation of the cellular cytosol, and contains, in addition to transcription and translation, a number of intact and functional core metabolic pathways (Kim and Swartz, 2001; Kim and Kim, 2009). Thus, cell-free systems have been successfully used as a prototyping platform for synthetic biology, a so-called "cellular breadboard"

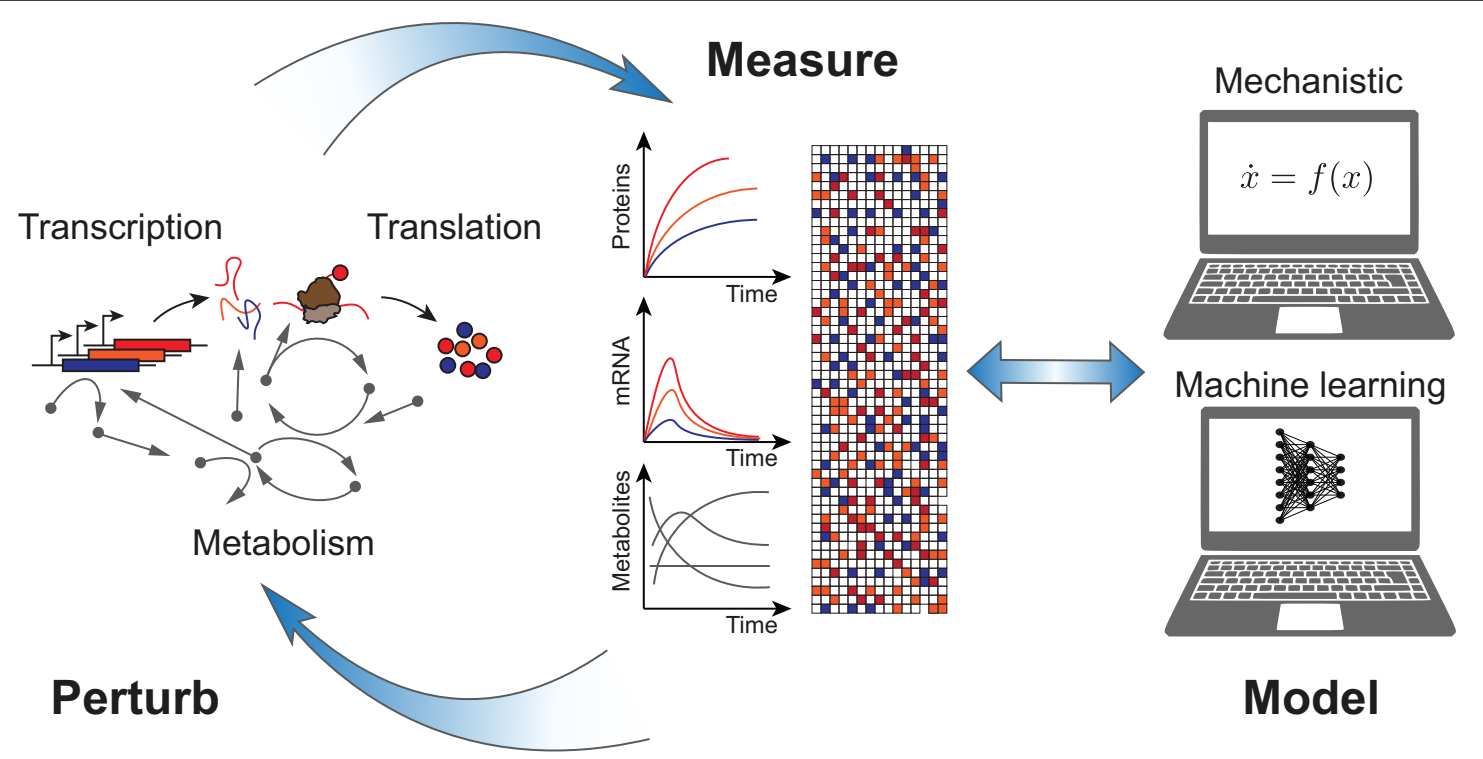

FIGURE 1 | Rational computer-aided biodesign with cell-free systems: cell-free gene expression systems perform in vitro transcription, translation, and metabolism in reactions which are open and transparent to measurement and perturbation. Data sets generated are kinetically resolved and high-throughput, facilitating development of both mechanistic as well as machine learning models. 
where synthetic gene circuit designs can be quickly implemented, validated, and ported back into a living cell (Siegal-Gaskins et al., 2014; Garamella et al., 2016). The success of this approach relies on a basic similarity between the cell-free and cellular environments, an assumption that has been verified in a number of notable examples (Chappell et al., 2013; Niederholtmeyer et al., 2015; Borkowski et al., 2018; Halleran and Murray, 2018; Hu et al., 2018).

On the other hand, cell-free systems do contain fundamental differences from cells. In addition to being non-living, there are a number of key biophysical differences. Below I highlight these, and consider their consequences in the context of the implementation of a generic synthetic gene regulatory network (GRN). In some cases, strategies for making the system more "lifelike" by bottom-up construction are briefly discussed.

1. Accessible system: Without a barrier between the reaction and the environment, the cell-free reaction is transparent to observation and perturbation, allowing the reaction conditions to be adjusted at will. This property can be leveraged, for example, to change redox environments to promote disulphide bond formation (Matsuda et al., 2013). The kinetic progress of reactions can be followed using fluorescence from proteins and mRNA, as well as realtime metabolomic profiling, which has allowed the internal metabolism of cell-free systems to be dissected at high resolution (Bujara et al., 2011; Vilkhovoy et al., 2019). For GRN design, parameters, such as dissociation and kinetic constants between a transcription factor and promoter may be measured in situ (Geertz et al., 2012; Swank et al., 2019), and perturbations applied to the reaction composition to facilitate parameter identification and model selection (Hu et al., 2015; Moore et al., 2018). These key properties of controllable inputs, perturbations, and consistency between conditions where parameter measurement and system operation take place directly address challenges faced in engineering living cells. Crucially, this enables a close coupling of cell-free experiments and computational models.

2. Dilute, well-mixed reaction environment: The lack of compartmentalization is also related to a number of other physical effects, including a loss of stochasticity, slower enzymatic rates, a reduced level of macromolecular crowding, a loss of spatial organization, and a loss of membraneassociated processes [although lysates can contain inverted membrane vesicles which permit oxidative phosphorylation (Jewett et al., 2008)]. A useful consequence of such a simplified reaction environment is that the system can be described with deterministic kinetics; a practical side-effect is that exogenous protein aggregation is minimized, which facilitates bioproduction. In order to recreate more lifelike reaction environments, there is much ongoing effort to encapsulate cell-free reactions in a variety of compartments including liposomes, polymersomes, and droplets, as well as introducing crowding and organization into cell-free systems (Laohakunakorn et al., 2020).

3. Relaxation to equilibrium: Living cells are maintained in a homeostatic, non-equilibrium steady state by a constant flux of energy and metabolites through the system, while cell-free reactions relax to biochemical equilibrium as the reaction proceeds. This sets a limit on the lifetime of the cellfree reaction. The lifetime may be extended by engineering a more homeostatic metabolic system [for instance, rationallydesigned in vitro metabolic systems can operate autonomously for days (Korman et al., 2017)], but ultimately to maintain cell-free systems in a steady state, an energy and metabolite flux must be set up between the system and environment. This can be achieved using continuous flow or continuous exchange reactors (Spirin et al., 1988; Niederholtmeyer et al., 2013; Karzbrun et al., 2014), or by compartmentalizing and coupling the reaction to transport processes (Noireaux and Libchaber, 2004). A consequence of limited lifetime is that after a few hours, any synthetic cell-free gene circuit ceases to be functional. Thus, recent efforts have focused on extending reaction lifetimes (Caschera and Noireaux, 2015) as well as accelerating the computational and output steps in the circuit (Alam et al., 2019).

4. No regulation: While living cells are actively regulated at multiple levels of organization, from molecularto network-scale, cell-free systems contain no active regulatory mechanisms. This simplifies the identification and measurement of host-chassis interactions, allowing resource allocation within the cell-free system to be elucidated in detail (Siegal-Gaskins et al., 2014; Gyorgy and Murray, 2016; Borkowski et al., 2018; Halter et al., 2018). On the other hand, cell-free systems lose the robustness conferred by homeostasis (Lewis et al., 2014). They are thus sensitive to effects which would otherwise be regulated, for example partial degradation products (Kim and Winfree, 2011), stochasticity in gene expression (Karig et al., 2013), and variable partitioning of reactants during system encapsulation (Altamura et al., 2018). This property may thus be an impediment to predictive design.

5. No self-regeneration: Self-regeneration is a defining hallmark of life (Luisi et al., 2006), and cell-free systems do not regenerate their components, implying that the lifetime of cellfree reactions is also limited by enzyme stability (Stögbauer et al., 2012), in addition to resource depletion and metabolic arrest. The possibility of programming regeneration directly in the cell-free system leads to the tantalizing prospect of a cell-free system capable of maintaining its components, which could form the basis of an engine to power artificial cells (Schwille et al., 2018).

6. No replication: In addition to not regenerating their components, cell-free systems also do not replicate their genetic material. This has been considered an opportunity for bottom-up reconstruction, from early demonstrations of in vitro replication of plasmids and viral DNA in prokaryotic and eukaryotic lysates (Diaz and Staudenbauer, 1982; Li and Kelly, 1984; Stillman and Gluzman, 1985) to more recent studies involving phi29 DNA polymerase (Sakatani et al., 2015; van Nies et al., 2018), which have culminated in the replication of up to $116 \mathrm{~kb}$ of DNA in the PURE system (Libicher et al., 2020). Lack of replication implies genetic stability of introduced DNA, unlike living cells which can 
mutate away exogenous gene circuit function. Steady-state in vitro replication of nucleic acids would form a necessary subsystem of self-replicating artificial cells as well as enable in vitro evolutionary studies (Meyer et al., 2012).

These properties have influenced the approaches used in cellfree engineering. In particular, the accessibility of the reaction environment has made cell-free systems particularly suited for rational biodesign strategies, as will be discussed next.

\section{RATIONAL BIODESIGN STRATEGIES FOR CELL-FREE SYNTHETIC BIOLOGY}

\subsection{Model-Guided Design}

The most ambitious approach to rational biodesign uses a quantitative and predictive model to guide the design process, adopting workflows from well-established fields, such as electrical and aerospace engineering. For synthetic biology, the largest obstacles to this involve unknown, uncharacterized, or changing interactions among biomolecular components, and the difficulty of accessing and perturbing system components. In general, we can envisage two broad approaches which aim to mitigate this knowledge gap in cell-free systems: a "bottom-up" approach, where purified, reconstituted systems are constructed one component at a time, allowing interactions to be taken into account as they arise; and a "top-down" approach, where crude cellular lysates are interrogated and potentially modified to remove unwanted interactions, exposing the minimal system beneath. These approaches mirror the bottom-up and top-down approaches to the construction of artificial cells, with the final result being a minimal system that is maximally understood.

Recently, efforts have been made to combine the development of reconstituted cell-free systems with mathematical modeling (Mavelli et al., 2015; Matsuura et al., 2017, 2018; Carrara et al., 2018; Doerr et al., 2019). Reconstituted systems are composed of purified cellular enzymes and an energy solution, mixed together in a known composition, and are available commercially [e.g., commonly-used variants based on the PURE system (Shimizu et al., 2001)]. Compared to lysates, reconstituted systems are dramatically simplified. In principle, since the exact system composition is known, a model incorporating all predicted interactions can be written down. In practice, it is infeasible to calibrate such fine-grained models to experimental measurements, although properties, such as robustness of the system can be investigated in silico (Matsuura et al., 2017). Current coarse-grained models are generally not sufficient to globally capture all observed experimental effects (Doerr et al., 2019). The overarching aim is therefore to search for computational models of appropriate granularity which can describe all experimental observations, and yet remain feasible for calibration. The success of this is likely to be borne out through approaches which combine automation and high-throughput measurements with improved cost-efficient methods for preparing recombinant systems (Lavickova and Maerkl, 2019).

The top-down, systems-level approach aims to develop mechanistic understanding by interrogating lysates, which contain significantly more unknowns. The 'black-box' of lysates has slowly been opened over the last two decades, motivated by a desire to improve productivity and lifetime of the system (Silverman et al., 2019b). Using a combination of strain engineering and data from biochemical and metabolic analyses, it is now possible to rationally redirect metabolic flux and energy usage. Energy regeneration schemes of increasing complexity have been developed in order to improve lysate reaction lifetime and yield, proceeding initially from single-step (Zubay, 1973; Kigawa et al., 1999) to multi-step pathways which regenerate ATP using enzymes present within the extract (Kim and Swartz, 1999, 2001; Jewett and Swartz, 2004; Sitaraman et al., 2004; Calhoun and Swartz, 2005; Jewett et al., 2008; Caschera and Noireaux, 2015). While the complexity of lysates is considerable, in contrast to cellular systems biology, cell-free systems are amenable to essentially unconstrained perturbation, which greatly facilitates model testing and validation. This has been demonstrated by a number of modeling studies of increasing sophistication (Karzbrun et al., 2011; Stögbauer et al., 2012; Tuza et al., 2015; Gyorgy and Murray, 2016; Nieß et al., 2017; Marshall and Noireaux, 2019), as well as notable examples of model-guided forward engineering of genetic circuits ( $\mathrm{Hu}$ et al., 2015, 2018; Agrawal et al., 2019; Lehr et al., 2019; Westbrook et al., 2019). Recent development of integrated gene expression and metabolic models have elucidated the factors limiting CFPS (Wayman et al., 2015; Vilkhovoy et al., 2018, 2019; Horvath et al., 2020), suggesting that combined computational and experimental metabolomic studies are poised to contribute significantly to our understanding of CFPS in lysates.

\subsection{Control Theoretic Approaches for Robust Operation}

Even if all interactions could be measured, many are unlikely to remain constant with time. Additionally, cell-free reactions operate dynamically, in an equally dynamic, fluctuating environment. It is clear, then, that knowledge of interactions is not generally sufficient to ensure robust performance.

Control engineering attempts to maintain the performance of a dynamic system within certain specified bounds, while parts of the system are subject to uncontrolled disturbances. A specific example of this is reference tracking using feedback control, where an output, such as the gene expression level follows a reference signal despite the presence of perturbations. Achieving this requires the system to sense the reference as well as its output, which involves redirecting the output back into the system in a feedback loop. Another example is buffering outputs against upstream variability using feed-forward regulators which balance each other's activity. Feedback and feedforward loops are ubiquitous in natural biological systems, making it a natural extension to develop synthetic biology within a control theoretic framework (Vecchio et al., 2016; Del Vecchio et al., 2018; Hsiao et al., 2018; Baetica et al., 2019).

Feedback control has been successfully implemented in a number of in vivo examples, for instance to regulate exogenous gene expression in response to burden (Ceroni et al., 2018), or to control growth rate using robust perfect adaptation (Aoki 
et al., 2019). Feedforward architectures have also been used to control variations in the amount of DNA template present in cells (Bleris et al., 2011). In the context of control circuits, a significant advantage of cell-free over cellular systems is that they are free from biological noise, and operate in the deterministic regime. Despite these benefits, surprisingly little work has been carried out on cell-free control; recent examples, including a computational study (Agrawal et al., 2018) and an experimental demonstration of a feedback integral controller based on molecular sequestration (Agrawal et al., 2019), as well as feedforward loop circuits (Guo and Murray, 2019), suggest that such approaches are starting to become more widespread within the cell-free community.

\subsection{Active Learning}

While a mechanistic or phenomenological model may lead to transparent understanding of the system, they are not the only models offering sufficient predictive capabilities for rational design. Statistical or non-parametric models, developed from data using machine learning approaches, can be equally or more strongly predictive, albeit with the well-known challenges of interpretability (Doshi-Velez and Kim, 2017).

An example problem is to determine the composition of a cell-free reaction to maximize its protein productivity. In the absence of a predictive model fully connecting all its components to protein output, Caschera et al. (2018) used an evolutionary design of experiments approach to iteratively train an ensemble neural network model to optimize conditions for cell-free protein synthesis. More recently Borkowski et al. (2020) trained a similar model on $\sim 4,000$ reactions, improving yields by 34 times. Importantly, they discovered a training dataset of only 20 compositions which was informative enough to allow the model to generalize its predictions to different lysates and conditions. These examples demonstrate that the throughput afforded by cell-free systems is sufficient for informing datadriven modeling approaches.

Data-driven cell-free techniques could also potentially be applied to other long-standing questions in systems biology, for instance, determining the mapping of sequence to phenotype for a genetic element (Cuperus et al., 2017; Sample et al., 2019). Cell-free implementations of massively parallel reporter assays, perhaps using droplet microfluidic technology to maintain genotype-phenotype linkage, may yield datasets of sufficient quality and size to contribute to this problem.

\section{REFERENCES}

Agrawal, D. K., Marshall, R., Noireaux, V., and Sontag, E. D. (2019). In vitro implementation of robust gene regulation in a synthetic biomolecular integral controller. Nat. Commun. 10, 1-12. doi: 10.1038/s41467-019-13626-z

Agrawal, D. K., Tang, X., Westbrook, A., Marshall, R., Maxwell, C. S., Lucks, J., et al. (2018). Mathematical modeling of RNA-based architectures for closed loop control of gene expression. ACS Synth. Biol. 7, 1219-1228. doi: 10.1021/acssynbio.8b00040

Alam, K. K., Jung, J. K., Verosloff, M. S., Clauer, P. R., Lee, J. W., Capdevila, D. A., et al. (2019). Rapid, low-cost detection of water contaminants using regulated in vitro transcription. biorXiv. doi: 10.1101/619296

\section{CONCLUSIONS}

Cell-free systems are ideally suited for rational engineering approaches: their open reactions facilitate construction and validation of mechanistic and phenomenological models, and their throughput allows them to generate sufficient data to train machine learning models. Developing these models, and designing experiments to calibrate and validate them, are general strategies which can be tested on the cell-free platform, but eventually also applied to the more challenging problem of engineering living systems. In this sense, cell-free systems can be thought of as a proving ground for rational design strategies.

Cell-free systems do however present unique challenges for predictive design. As discussed above, a lack of homeostasis can imply ultrasensitivity of cell-free reactions to various effects. It is also well-known that strong batch-to-batch variation of lysates can limit the predictability of results to within-batch repeats, constraining the usefulness of the approach; however recent efforts have been made to identify and control these effects (Cole et al., 2019; Silverman et al., 2019b). And finally, while examples were given of successful transfer of cell-free designs back into cellular hosts, the generality of this approach has so far remained unclear. These are all avenues for future research within the field.

In this Perspective, I have deliberately left out a discussion of directed evolution, a complementary and powerful strategy for biodesign. Cell-free systems have also been extensively deployed for in vitro evolution (Contreras-Llano and Tan, 2018), maintaining genotype-phenotype coupling through the use of display technologies and compartmentalization.

Reliable engineering of synthetic biological systems remains a great challenge, and it is likely that a number of complementary efforts including rational as well as evolutionary design, and cellular and cell-free systems, will be required to eventually achieve this grand goal.

\section{AUTHOR CONTRIBUTIONS}

NL conceived and wrote the article.

\section{FUNDING}

NL was supported by a Chancellor's Fellowship from the University of Edinburgh. 
Baetica, A.-A., Westbrook, A., and El-Samad, H. (2019). Control theoretical concepts for synthetic and systems biology. Curr. Opin. Syst. Biol. 14, 50-57. doi: 10.1016/j.coisb.2019.02.010

Bleris, L., Xie, Z., Glass, D., Adadey, A., Sontag, E., and Benenson, Y. (2011). Synthetic incoherent feedforward circuits show adaptation to the amount of their genetic template. Mol. Syst. Biol. 7, 1-12. doi: 10.1038/msb.2011.49

Borkowski, O., Bricio, C., Murgiano, M., Rothschild-Mancinelli, B., Stan, G.-B., and Ellis, T. (2018). Cell-free prediction of protein expression costs for growing cells. Nat. Commun. 9, 1-11. doi: 10.1038/s41467-018-03970-x

Borkowski, O., Koch, M., Zettor, A., Pandi, A., Batista, A. C., Soudier, P., et al. (2020). Large scale active-learning-guided exploration for in vitro protein production optimization. Nat. Commun. 11, 1-8. doi: 10.1038/s41467-020-15798-5

Bujara, M., Schümperli, M., Pellaux, R., Heinemann, M., and Panke, S. (2011). Optimization of a blueprint for in vitro glycolysis by metabolic real-time analysis. Nat. Chem. Biol. 7, 271-277. doi: 10.1038/nchembio.541

Calhoun, K. A., and Swartz, J. R. (2005). Energizing cell-free protein synthesis with glucose metabolism. Biotechnol. Bioeng. 90, 606-613. doi: 10.1002/bit.20449

Canton, B., Labno, A., and Endy, D. (2008). Refinement and standardization of synthetic biological parts and devices. Nat. Biotechnol. 26, 787-793. doi: $10.1038 /$ nbt1413

Carrara, P., Altamura, E., D'Angelo, F., Mavelli, F., and Stano, P. (2018). Measurement and numerical modeling of cell-free protein synthesis: combinatorial block-variants of the PURE system. Data 3, 1-12. doi: 10.3390/data3040041

Caschera, F., Karim, A. S., Gazzola, G., D’Aquino, A. E., Packard, N. H., and Jewett, M. C. (2018). High-throughput optimization cycle of a cell-free ribosome assembly and protein synthesis system. ACS Synth. Biol. 7, 28412853. doi: 10.1021 /acssynbio. 8 b00276

Caschera, F., and Noireaux, V. (2015). Preparation of amino acid mixtures for cell-free expression systems. Biotechniques 58, 40-42. doi: 10.2144/000114249

Ceroni, F., Boo, A., Furini, S., Gorochowski, T. E., Borkowski, O., Ladak, Y. N., et al. (2018). Burden-driven feedback control of gene expression. Nat. Methods 15, 387-393. doi: 10.1038/nmeth.4635

Chappell, J., Jensen, K., and Freemont, P. S. (2013). Validation of an entirely in vitro approach for rapid prototyping of DNA regulatory elements for synthetic biology. Nucleic Acids Res. 41, 3471-3481. doi: 10.1093/nar/gkt052

Cole, S. D., Beabout, K., Turner, K. B., Smith, Z. K., Funk, V. L., Harbaugh, S. V., et al. (2019). Quantification of interlaboratory cell-free protein synthesis variability. ACS Synth. Biol. 8, 2080-2091. doi: 10.1021/acssynbio.9b00178

Contreras-Llano, L. E., and Tan, C. (2018). High-throughput screening of biomolecules using cell-free gene expression systems. Synth. Biol. 3, 1-13. doi: 10.1093/synbio/ysy012

Cuperus, J. T., Groves, B., Kuchina, A., Rosenberg, A. B., Jojic, N., Fields, S., et al. (2017). Deep learning of the regulatory grammar of yeast 5' untranslated regions from 500,000 random sequences. Genome Res. 27, 20152024. doi: $10.1101 /$ gr.224964.117

Davies, J. A. (2019). Real-world synthetic biology: is it founded on an engineering approach, and should it be? Life 9, 1-15. doi: 10.3390/life9010006

Del Vecchio, D., Qian, Y., Murray, R. M., and Sontag, E. D. (2018). Future systems and control research in synthetic biology. Annu. Rev. Control 45, 5-17. doi: 10.1016/j.arcontrol.2018.04.007

Diaz, R., and Staudenbauer, W. L. (1982). Replication of the broad host range plasmid RSF1010 in cell-free extracts of Escherichia coli and Pseudomonas aeruginosa. Nucleic Acids Res. 10, 4687-4702. doi: 10.1093/nar/10.15.4687

Doerr, A., de Reus, E., van Nies, P., van der Haar, M., Wei, K., Kattan, J., et al. (2019). Modelling cell-free RNA and protein synthesis with minimal systems. Phys. Biol. 16:025001. doi: 10.1088/1478-3975/aaf33d

Doshi-Velez, F., and Kim, B. (2017). Towards a rigorous science of interpretable machine learning. arXiv [Preprint] arXiv:1702.08608.

Endy, D. (2005). Foundations for engineering biology. Nature 438, 449-453. doi: 10.1038 /nature 04342

Garamella, J., Marshall, R., Rustad, M., and Noireaux, V. (2016). The all E. coli TXTL toolbox 2.0: a platform for cell-free synthetic biology. ACS Synth. Biol. 5, 344-355. doi: 10.1021/acssynbio.5b00296

Garenne, D., and Noireaux, V. (2019). Cell-free transcription-translation: engineering biology from the nanometer to the millimeter scale. Curr. Opin. Biotechnol. 58, 19-27. doi: 10.1016/j.copbio.2018.10.007
Geertz, M., Shore, D., and Maerkl, S. J. (2012). Massively parallel measurements of molecular interaction kinetics on a microfluidic platform. Proc. Natl. Acad. Sci. U.S.A. 109, 16540-16545. doi: 10.1073/pnas.1206011109

Gregorio, N. E., Levine, M. Z., Oza, J. P., Gregorio, N. E., Levine, M. Z., and Oza, J. P. (2019). A user's guide to cell-free protein synthesis. Methods Protoc. 2:24. doi: $10.3390 / \mathrm{mps} 2010024$

Guo, S., and Murray, R. M. (2019). Construction of incoherent feedforward loop circuits in a cell-free system and in cells. ACS Synth. Biol. 8, 606-610. doi: 10.1021/acssynbio.8b00493

Gyorgy, A., and Murray, R. M. (2016). Quantifying resource competition and its effects in the TX-TL system, in 2016 IEEE 55th Conference on Decision and Control, CDC 2016, Vol. 1 (Las Vegas, NV), 3363-3368. doi: 10.1109/CDC.2016.7798775

Halleran, A. D., and Murray, R. M. (2018). Cell-free and in vivo characterization of Lux, Las, and Rpa quorum activation systems in E. coli. ACS Synth. Biol. 7, 752-755. doi: 10.1021/acssynbio.7b00376

Halter, W., Allgower, F., Murray, R. M., and Gyorgy, A. (2018). Optimal experiment design and leveraging competition for shared resources in cellfree extracts, in 2018 IEEE Conference on Decision and Control (CDC) (Miami Beach, FL: IEEE), 1872-1879. doi: 10.1109/CDC.2018.8619039

Heinemann, M., and Panke, S. (2006). Synthetic biology-putting engineering into biology. Bioinformatics 22, 2790-2799. doi: 10.1093/bioinformatics/btl469

Hillson, N., Caddick, M., Cai, Y., Carrasco, J. A., Chang, M. W., Curach, N. C., et al. (2019). Building a global alliance of biofoundries. Nat. Commun. 10, 1-4. doi: 10.1038/s41467-019-10079-2

Horvath, N., Vilkhovoy, M., Wayman, J. A., Calhoun, K., Swartz, J., and Varner, J. D. (2020). Toward a genome scale sequence specific dynamic model of cellfree protein synthesis in Escherichia coli. Metab. Eng. Commun. 10:e00113. doi: 10.1016/j.mec.2019.e00113

Hsiao, V., Swaminathan, A., and Murray, R. M. (2018). Control theory for synthetic biology. IEEE Control Syst. 38, 32-62. doi: 10.1109/MCS.2018.2810459

Hu, C. Y., Takahashi, M. K., Zhang, Y., and Lucks, J. B. (2018). Engineering a functional small RNA negative autoregulation network with model-guided design. ACS Synth. Biol. 7, 1507-1518. doi: 10.1021/acssynbio.7b00440

Hu, C. Y., Varner, J. D., and Lucks, J. B. (2015). Generating effective models and parameters for RNA genetic circuits. ACS Synth. Biol. 4, 914-926. doi: 10.1021/acssynbio.5b00077

Jewett, M. C., Calhoun, K. A., Voloshin, A., Wuu, J. J., and Swartz, J. R. (2008). An integrated cell-free metabolic platform for protein production and synthetic biology. Mol. Syst. Biol. 4, 1-10. doi: 10.1038/msb.2008.57

Jewett, M. C., and Swartz, J. R. (2004). Mimicking the Escherichia coli cytoplasmic environment activates long-lived and efficient cell-free protein synthesis. Biotechnol. Bioeng. 86, 19-26. doi: 10.1002/bit.20026

Karig, D. K., Jung, S. Y., Srijanto, B., Collier, C. P., and Simpson, M. L. (2013). Probing cell-free gene expression noise in femtoliter volumes. ACS Synth. Biol. 2, 497-505. doi: 10.1021/sb400028c

Karim, A. S., and Jewett, M. C. (2018). Cell-free synthetic biology for pathway prototyping. Methods Enzymol. 608, 31-57. doi: 10.1016/bs.mie.2018.04.029

Karzbrun, E., Shin, J., Bar-Ziv, R. H., and Noireaux, V. (2011). Coarse-grained dynamics of protein synthesis in a cell-free system. Phys. Rev. Lett. 106, 1-4. doi: 10.1103/PhysRevLett.106.048104

Karzbrun, E., Tayar, A. M., Noireaux, V., and Bar-Ziv, R. H. (2014). Programmable on-chip DNA compartments as artificial cells. Science 345, 829-832. doi: $10.1126 /$ science. 1255550

Kigawa, T., Yabuki, T., Yoshida, Y., Tsutsui, M., Ito, Y., Shibata, T., et al. (1999). Cell-free production and stable-isotope labeling of milligram quantities of proteins. FEBS Lett. 442, 15-19. doi: 10.1016/S0014-5793(98)01620-2

Kim, D. M., and Swartz, J. R. (1999). Prolonging cell-free protein synthesis with a novel ATP regeneration system. Biotechnol. Bioeng. 66, 180-188. doi: 10.1002/(SICI) 1097-0290(1999)66:3<180::AID-BIT6>3.0.CO;2-S

Kim, D. M., and Swartz, J. R. (2001). Regeneration of adenosine triphosphate from glycolytic intermediates for cell-free protein synthesis. Biotechnol. Bioeng. 74, 309-316. doi: 10.1002/bit.1121

Kim, H.-C., and Kim, D.-M. (2009). Methods for energizing cell-free protein synthesis. J. Biosci. Bioeng. 108, 1-4. doi: 10.1016/j.jbiosc.2009.02.007

Kim, J., and Winfree, E. (2011). Synthetic in vitro transcriptional oscillators. Mol. Syst. Biol. 7, 1-15. doi: 10.1038/msb.2010.119 
Korman, T. P., Opgenorth, P. H., and Bowie, J. U. (2017). A synthetic biochemistry platform for cell free production of monoterpenes from glucose. Nat. Commun. 8, 1-8. doi: 10.1038/ncomms15526

Laohakunakorn, N., Grasemann, L., Lavickova, B., Michielin, G., Shahein, A., Swank, Z., et al. (2020). Bottom-up construction of complex biomolecular systems with cell-free synthetic biology. Front. Bioeng. Biotechnol. 8:213. doi: 10.3389/fbioe.2020.00213

Lavickova, B., and Maerkl, S. J. (2019). A simple, robust, and low-cost method to produce the PURE cell-free system. ACS Synth. Biol. 8, 455-462. doi: $10.1021 /$ acssynbio.8b00427

Lehr, F. X., Hanst, M., Vogel, M., Kremer, J., Göringer, H. U., Suess, B., et al. (2019). Cell-free prototyping of AND-logic gates based on heterogeneous RNA activators. ACS Synth. Biol. 8, 2163-2173. doi: 10.1021/acssynbio.9b00238

Lewis, D. D., Villarreal, F. D., Wu, F., and Tan, C. (2014). Synthetic biology outside the cell: linking computational tools to cell-free systems. Front. Bioeng. Biotechnol. 2:66. doi: 10.3389/fbioe.2014.00066

Li, J. J., and Kelly, T. K. (1984). Simian virus 40 DNA replication in vitro. Proc. Natl. Acad. Sci. U.S.A. 81, 6973-6977. doi: 10.1073/pnas.81.22.6973

Libicher, K., Hornberger, R., Heymann, M., and Mutschler, H. (2020). In vitro self-replication and multicistronic expression of large synthetic genomes. Nat. Commun. 11:904. doi: 10.1038/s41467-020-14694-2

Luisi, P. L., Ferri, F., and Stano, P. (2006). Approaches to semi-synthetic minimal cells: a review. Naturwissenschaften 93, 1-13. doi: 10.1007/s00114-005-0056-Z

MacDonald, J. T., Barnes, C., Kitney, R. I., Freemont, P. S., and Stan, G. B. V. (2011). Computational design approaches and tools for synthetic biology. Integr. Biol. 3, 97-108. doi: 10.1039/c0ib00077a

Marshall, R., and Noireaux, V. (2019). Quantitative modeling of transcription and translation of an all-E. coli cell-free system. Sci. Rep. 9, 1-12. doi: 10.1038/s41598-019-48468-8

Matsuda, T., Watanabe, S., and Kigawa, T. (2013). Cell-free synthesis system suitable for disulfide-containing proteins. Biochem. Biophys. Res. Commun. 431, 296-301. doi: 10.1016/j.bbrc.2012.12.107

Matsuura, T., Hosoda, K., and Shimizu, Y. (2018). Robustness of a reconstituted Escherichia coli protein translation system analyzed by computational modeling. ACS Synth. Biol. 7, 1964-1972. doi: 10.1021/acssynbio.8b00228

Matsuura, T., Tanimura, N., Hosoda, K., Yomo, T., and Shimizu, Y. (2017). Reaction dynamics analysis of a reconstituted Escherichia coli protein translation system by computational modeling. Proc. Natl. Acad. Sci. U.S.A. 114, E1336-E1344. doi: 10.1073/pnas.1615351114

Mavelli, F., Marangoni, R., and Stano, P. (2015). A simple protein synthesis model for the PURE system operation. Bull. Math. Biol. 77, 1185-1212. doi: 10.1007/s11538-015-0082-8

Meyer, A. J., Ellefson, J. W., and Ellington, A. D. (2012). Abiotic self-replication. Acc. Chem. Res. 45, 2097-2105. doi: 10.1021/ar200325v

Moore, S. J., MacDonald, J. T., Wienecke, S., Ishwarbhai, A., Tsipa, A., Aw, R., et al. (2018). Rapid acquisition and model-based analysis of cell-free transcriptiontranslation reactions from nonmodel bacteria. Proc. Natl. Acad. Sci. U.S.A. 115, E4340-E4349. doi: 10.1073/pnas.1715806115

Niederholtmeyer, H., Stepanova, V., and Maerkl, S. J. (2013). Implementation of cell-free biological networks at steady state. Proc. Natl. Acad. Sci. U.S.A. 110, 15985-15990. doi: 10.1073/pnas.1311166110

Niederholtmeyer, H., Sun, Z. Z., Hori, Y., Yeung, E., Verpoorte, A., Murray, R. M., et al. (2015). Rapid cell-free forward engineering of novel genetic ring oscillators. eLife 4, 1-18. doi: 10.7554/eLife.09771

Nielsen, A. A., Der, B. S., Shin, J., Vaidyanathan, P., Paralanov, V., Strychalski, E. A., et al. (2016). Genetic circuit design automation. Science 352:aac7341. doi: $10.1126 /$ science.aac7341

Nieß, A., Failmezger, J., Kuschel, M., Siemann-Herzberg, M., and Takors, R. (2017). Experimentally validated model enables debottlenecking of in vitro protein synthesis and identifies a control shift under in vivo conditions. ACS Synth. Biol. 6, 1913-1921. doi: 10.1021/acssynbio.7b00117

Nirenberg, M. W., and Matthaei, J. H. (1961). The dependence of cell-free protein synthesis in E. coli upon naturally occurring or synthetic polyribonucleotides. Proc. Natl. Acad. Sci. U.S.A. 47, 1588-1602. doi: 10.1073/pnas.47.10.1588

Noireaux, V., and Libchaber, A. (2004). A vesicle bioreactor as a step toward an artificial cell assembly. Proc. Natl. Acad. Sci. U.S.A. 101, 17669-17674. doi: $10.1073 /$ pnas. 0408236101
Pardee, K., Green, A. A., Ferrante, T., Cameron, D. E., Daleykeyser, A., Yin, P., et al. (2014). Paper-based synthetic gene networks. Cell 159, 940-954. doi: 10.1016/j.cell.2014.10.004

Perez, J. G., Stark, J. C., and Jewett, M. C. (2016). Cell-free synthetic biology: engineering beyond the cell. Cold Spring Harbor Perspect. Biol. 8:a023853. doi: $10.1101 /$ cshperspect.a023853

Sakatani, Y., Ichihashi, N., Kazuta, Y., and Yomo, T. (2015). A transcription and translation-coupled DNA replication system using rolling-circle replication. Sci. Rep. 5, 1-5. doi: 10.1038/srep10404

Sample, P. J., Wang, B., Reid, D. W., Presnyak, V., McFadyen, I. J., Morris, D. R., et al. (2019). Human 5' UTR design and variant effect prediction from a massively parallel translation assay. Nat. Biotechnol. 37, 803-809. doi: 10.1038/s41587-019-0164-5

Schwille, P., Spatz, J., Landfester, K., Herminghaus, S., Sourjik, V., Erb, T. J., et al. (2018). MaxSynBio: avenues towards creating cells from the bottom up. Angew. Chem. Int. Ed. 57, 13382-13392. doi: 10.1002/anie.201802288

Shimizu, Y., Inoue, A., Tomari, Y., Suzuki, T., Yokogawa, T., Nishikawa, K., et al. (2001). Cell-free translation reconstituted with purified components. Nat. Biotechnol. 19, 751-755. doi: 10.1038/90802

Siegal-Gaskins, D., Tuza, Z. A., Kim, J., Noireaux, V., and Murray, R. M. (2014). Gene circuit performance characterization in a cell-free 'breadboard'. ACS Synth. Biol. 3, 416-425. doi: 10.1021/sb400203p

Silverman, A. D., Karim, A. S., and Jewett, M. C. (2019a). Cell-free gene expression: an expanded repertoire of applications. Nat. Rev. Genet. 21, 151170. doi: 10.1038/s41576-019-0186-3

Silverman, A. D., Kelley-Loughnane, N., Lucks, J. B., and Jewett, M. C. (2019b). Deconstructing cell-free extract preparation for in vitro activation of transcriptional genetic circuitry. ACS Synth. Biol. 8, 403-414. doi: 10.1021/acssynbio.8b00430

Sitaraman, K., Esposito, D., Klarmann, G., Le Grice, S. F., Hartley, J. L., and Chatterjee, D. K. (2004). A novel cell-free protein synthesis system. J. Biotechnol. 110, 257-263. doi: 10.1016/j.jbiotec.2004.02.014

Spirin, A. S., Baranov, V. I., Ryabova, L. A., Ovodov, S. Y., and Alakhov, Y. B. (1988). A continous cell-free translation system capable of producing polypeptides in high yield. Science 242, 1162-1164. doi: 10.1126/science.3055301

Stillman, B. W., and Gluzman, Y. (1985). Replication and supercoiling of simian virus 40 DNA in cell extracts from human cells. Mol. Cell. Biol. 5, 2051-2060. doi: 10.1128/MCB.5.8.2051

Stögbauer, T., Windhager, L., Zimmer, R., and Rädler, J. O. (2012). Experiment and mathematical modeling of gene expression dynamics in a cell-free system. Integr. Biol. 4, 494-501. doi: 10.1039/c2ib00102k

Swank, Z., Laohakunakorn, N., and Maerkl, S. J. (2019). Cell-free gene-regulatory network engineering with synthetic transcription factors. Proc. Natl. Acad. Sci. U.S.A. 116, 5892-5901. doi: 10.1073/pnas. 1816591116

Takahashi, M. K., Hayes, C. A., Chappell, J., Sun, Z. Z., Murray, R. M., Noireaux, V., et al. (2015). Characterizing and prototyping genetic networks with cell-free transcription-translation reactions. Methods 86, 60-72. doi: 10.1016/j.ymeth.2015.05.020

Tuza, Z. A., Siegal-Gaskins, D., Kim, J., and Szederkenyi, G. (2015). Analysisbased parameter estimation of an in vitro transcription-translation system. Eur. Control Conf. 2015, 1554-1560. doi: 10.1109/ECC.2015.7330760

van Nies, P., Westerlaken, I., Blanken, D., Salas, M., Mencía, M., and Danelon, C. (2018). Self-replication of DNA by its encoded proteins in liposomebased synthetic cells. Nat. Commun. 9:1583. doi: 10.1038/s41467-01803926-1

Vecchio, D. D., Dy, A. J., and Qian, Y. (2016). Control theory meets synthetic biology. J. R. Soc. Interface 13:20160380. doi: 10.1098/rsif.2016. 0380

Vilkhovoy, M., Dai, D., Vadhin, S., Adhikari, A., and Varner, J. D. (2019). Absolute quantification of cell-free protein synthesis metabolism by reversedphase liquid chromatography-mass spectrometry. J. Vis. Exp. 152:e60329. doi: $10.3791 / 60329$

Vilkhovoy, M., Horvath, N., Shih, C. H., Wayman, J. A., Calhoun, K., Swartz, J., et al. (2018). Sequence specific modeling of E. coli cell-free protein synthesis. ACS Synth. Biol. 7, 1844-1857. doi: 10.1021/acssynbio. $7 \mathrm{~b} 00465$ 
Wayman, J. A., Sagar, A., and Varner, J. D. (2015). Dynamic modeling of cellfree biochemical networks using effective kinetic models. Processes 3, 138-160. doi: $10.3390 /$ pr3010138

Westbrook, A., Tang, X., Marshall, R., Maxwell, C. S., Chappell, J., Agrawal, D. K., et al. (2019). Distinct timescales of RNA regulators enable the construction of a genetic pulse generator. Biotechnol. Bioeng. 116, 1139-1151. doi: 10.1002/bit.26918

Zubay, G. (1973). In vitro synthesis of protein in microbial systems. Annu. Rev. Genet. 7, 267-287. doi: 10.1146/annurev.ge.07.120173. 001411
Conflict of Interest: The author declares that the research was conducted in the absence of any commercial or financial relationships that could be construed as a potential conflict of interest.

Copyright (c) 2020 Laohakunakorn. This is an open-access article distributed under the terms of the Creative Commons Attribution License (CC BY). The use, distribution or reproduction in other forums is permitted, provided the original author(s) and the copyright owner(s) are credited and that the original publication in this journal is cited, in accordance with accepted academic practice. No use, distribution or reproduction is permitted which does not comply with these terms. 